\title{
BMJ Open What does my neighbourhood have to do with my weight? A protocol for systematic review and meta-analysis of the association between neighbourhood socioeconomic status and body weight
}

\author{
Shimels Hussien Mohammed, ${ }^{1}$ Mulugeta Molla Birhanu, ${ }^{2}$ Tesfamichael Awoke \\ Sissay, ${ }^{2}$ Tesfa Dejenie Habtewold, ${ }^{2,3}$ Balewgizie Sileshi Tegegn, ${ }^{3}$ \\ Ahmad Esmaillzadeh ${ }^{4,5,6}$
}

To cite: Mohammed SH, Birhanu MM,

Sissay $T A$, et al. What does my neighbourhood have to do with my weight? A protocol for systematic review and metaanalysis of the association between neighbourhood socioeconomic status and body weight. BMJ Open 2017;7:e017567. doi:10.1136/ bmjopen-2017-017567

- Prepublication history and additional material for this paper are available online. To view please visit the journal (http:// dx.doi.org/10.1136/bmjopen2017-017567).

Received 2 May 2017 Revised 7 August 2017 Accepted 8 August 2017

\section{(a) CrossMark}

For numbered affiliations see end of article.

Correspondence to

Professor Ahmad Esmaillzadeh; a.esmaillzadeh@gmail.com

\section{ABSTRACT}

Introduction Individuals living in poor neighbourhoods are at a higher risk of overweight/obesity. There is no systematic review and meta-analysis study on the association of neighbourhood socioeconomic status (NSES) with overweight/obesity. We aimed to systematically review and meta-analyse the existing evidence on the association of NSES with overweight/obesity.

Methods and analysis Cross-sectional, case-control and cohort studies published in English from inception to 15 May 2017 will be systematically searched using the following databases: PubMed, EMBASE, Web of Sciences and Google Scholar. Selection, screening, reviewing and data extraction will be done by two reviewers, independently and in duplicate. The Newcastle-0ttawa Scale (NOS) will be used to assess the quality of evidence. Publication bias will be checked by visual inspection of funnel plots and Egger's regression test. Heterogeneity will be checked by Higgins's method ( ${ }^{2}$ statistics). Metaanalysis will be done to estimate the pooled OR. Narrative synthesis will be performed if meta-analysis is not feasible due to high heterogeneity of studies.

Ethics and dissemination Ethical clearance is not required as we will be using data from published articles. Findings will be communicated through a publication in a peer-reviewed journal and presentations at professional conferences.

PROSPERO registration number CRD42017063889.

\section{INTRODUCTION}

Obesity is a global public health threat, with negative consequences at both individual and societal levels. ${ }^{1}$ Over the past three decades there has been an enormous increase in the prevalence of overweight or obesity. ${ }^{12}$ Worldwide, $39 \%$ of adults aged 18 years and above were overweight in 2014. In the same year, $13 \%$ of adults were obese; more than double the figure in $1980 .^{3}$ Consecutive generations are at a higher risk of developing obesity at
Strengths and limitations of this study

- To the best of our knowledge, this is the first systematic review and meta-analysis to examine the association between NSES and overweight/obesity.

- The study will have wider representativeness, including individuals of all age groups and from all continents.

- The lack of uniformity in measures of NSES may yield significant heterogeneity and undermine comparability of studies.

an early age and at a higher rate. ${ }^{4}$ The WHO has adopted the prevention and control of obesity as a priority agenda and recommends nations to make a substantial improvement with regard to the current trend. ${ }^{5}$

Obesity has multiple risk factors, including those related to the environment. ${ }^{6} 7$ The neighbourhood in which individuals live has a strong influence on one's choice and adoption of health-enhancing behaviours and, consequently, the development of overweight/ obesity. ${ }^{89}$ Studies have shown that, irrespective of individual-level socioeconomic status, people living in poor neighbourhoods are more likely to be overweight/obese compared with those living in better-off neighbourhoods. ${ }^{10-12}$ Availability, access and utilisation of health-enhancing options are often limited in poor neighbourhoods, while fast food, alcohol and drugs are readily available. ${ }^{13}$ The walkability and safety of streets are often compromised, thus limiting the movement of residents. ${ }^{13} 14$ Poor neighbourhood socioeconomic conditions have also been linked to stress and depression, which are also risks for obesity. ${ }^{15}$

Investigating environmental drivers of obesity has increased recently. ${ }^{16} \mathrm{~A}$ number 
of studies were conducted and showed important results, including how poor neighbourhoods promote obesogenic lifestyles and result in a higher proportion of overweight/ obese individuals. ${ }^{10-14}$ However, there is variation in the studies, including population groups studied, geographical coverage and measures of neighbourhood socioeconomic status (NSES). To the best of our knowledge, there has been no systematic review and meta-analysis of studies reporting on the relationship between NSES and overweight/obesity. We believe synthesising these studies may fill the gap in the literature and provide stronger evidence for policy-making as there is an increasing recognition of systematic review and meta-analysis findings in the policy-making process. Thus, we will systematically review and meta-analyse studies that reported on the association of NSES with overweight/ obesity. The study will be comprehensive in terms of both geographical coverage and population groups addressed, such that studies across all continents and individuals of different age groups will be included.

\section{METHODS}

We will undertake systematic review and meta-analysis to answer three linked research questions:

1. What is the existing evidence on the risk of overweight/ obesity associated with NSES?

2. How do neighbourhood socioeconomic conditions influence overweight/obesity (mediating factors)?

3. What are the population groups who are more vulnerable to the obesogenic influences of poor NSES (moderating factors)?

\section{Protocol registration and reporting}

This protocol is registered in PROSPERO, registration number CRD42017063889. This protocol reporting follows the recommendations of the Meta-analysis of Observational Studies in Epidemiology (MOOSE) guideline ${ }^{17}$ and the Preferred Reporting Items for Systematic Reviews and Meta-Analyses Protocol (PRISMA-P) statement. ${ }^{18}$ The review process will follow the approach specified in this protocol. The results will be reported based on the PRISMA statement and MOOSE guidelines.

\section{Eligibility}

All observational studies (cross-sectional, case-control and cohort) will be included, provided they reported the association of NSES with weight status, which includes weight change, overweight, obesity or body mass index (BMI). Report of the studies (at least the abstract) should be in the English language. We will include all studies published from inception to 15 May 2017.

\section{Data source and search strategy}

A systematic literature search will be done using four databases: PubMed, EMBASE, Web of Sciences and Google Scholar. The search will be done using a combination of free texts and MeSH terms. In consultation with an experienced librarian, the search model was developed for
PubMed (see online supplementary file 1 ). The model will be adopted to the other databases. The database search will be supplemented by hand searching reference lists of identified articles.

\section{Inclusion and exclusion criteria}

We will include studies in which the association of NSES with overweight/obesity was reported irrespective of sex, age, geographical location and study year. The main outcome of interest is overweight/obesity, reported by BMI or related indices. The main exposure of interest is NSES, measured by composite indices like NSES, neighbourhood deprivation or neighbourhood economic hardship indices. Articles will be excluded on any one of the following conditions: (1) focused primarily on physical, policy or social environment; (2) language other than English; (3) no full text; or (4) qualitative studies, book chapters, symposium and conference proceedings, essays, commentaries, editorials and case reports.

\section{Study selection}

Articles from the database searches will be imported into RefWorks version 2.0 programme (http://www.refworks. com) and de-duplicated. To determine eligibility for fulltext screening, the title and abstract of the studies will be screened by two reviewers independently. Disagreement on eligibility will be resolved by consensus. After consensus is reached, the full text of eligible articles will be retrieved for an extended review and screened by SHM and MMB, independently and in duplicate. The screening and selection process of reviewed articles will be illustrated using a PRISMA flow diagram (see online supplementary file 2).

\section{Data abstraction}

Two review authors (SHM and TDH) will extract data independently and in duplicate. Discrepancies will be resolved by consensus. We will use a standardised format (see online supplementary file 3 ) to extract the following information from each eligible article:

1. Study identification (title, first author, year of publication)

2. Study characteristics (country/region, study design, sample size, follow-up period for longitudinal studies)

3. Participants' demographic factors (mean age, proportion of men)

4. NSES measurement method and category of NSES

5. Weight status (indices and mean value)

6. Measures of association between NSES and weight status (RR/OR with $95 \% \mathrm{CI})$

7. Information on adjustments (multivariate analyses, confounding variables)

\section{Assessment of study quality}

All included studies will be assessed by two reviewers (SHM, TDH) independently and in duplicate. In grading the quality of studies we will be guided by the NewcastleOttawa Scale (NOS). ${ }^{19}$ Ratings for each study will be 
compared between the two evaluators and discrepancies, if any, will be resolved by consensus.

\section{Statistical analysis}

We will check funnel plots to investigate publication bias. If the funnel plots show asymmetry, Egger's regression test will be performed. ${ }^{20}$ Publication bias will be assumed at $p$ value $<0.1$. If we find publication bias, we will apply the Duval and Tweedie trim-and-fill method. ${ }^{21}$ This method is based on the assumption that the effect sizes of the studies are normally distributed around the centre of the funnel plotthat is, in the event of asymmetries, it adjusts for the potential effect of unpublished studies. Heterogeneity among studies will be assessed by the $\mathrm{X}^{2}$ test for the Cochran's $\mathrm{Q}$ and $\mathrm{I}^{2}$ statistics, an estimate of the proportion of variance explained by between-study heterogeneity. An $\mathrm{I}^{2}$ value less than $50 \%$ represents a non-substantial level of heterogeneity. ${ }^{22}$ We will do separate analyses for low-, middle- and high-income countries. Subgroup analyses will also be done by age, gender, BMI levels or the types of NSES measurement methods used in the studies which may include NSES, neighbourhood deprivation or neighbourhood economic hardship indices. Studies will be presented as a summary table and forest plot(s). Meta-analysis will be done to pool estimates (OR/RR, 95\% CI). Narrative synthesis will be performed if meta-analysis is not feasible due to high heterogeneity of studies. Assuming there will be variation in the studies, we will use random effect and weighting in determining pooled estimates. Review Manager (RevMan) version 5.3.5 (Cochrane Informatics and Knowledge Management Department) for Windows ${ }^{23}$ will be used for all analyses.

\section{DISCUSSION}

The findings of this study will have important policy implications. By pooling the existing evidence on the link of NSES with overweight/obesity, we will provide a strong evidence base for decision making. We will also synthesise and report the potential moderating and mediating factors in the association, thereby showing the population segments who are more vulnerable to the obesogenic influences of neighbourhood deprivation. In these ways, we will highlight the need for comprehensive obesity prevention and control strategies, including addressing NSES disparities. The current approaches are mainly focused on addressing individual-level behavioural risks. We believe that addressing NSES disparities by bringing healthy choices closer to everyone may facilitate the adoption of health-enhancing behaviours.

We anticipate some potential limitations and strengths in our study. The inclusion of observational studies will preclude making a causal inference as reverse causality could not be ruled out. The lack of uniformity in NSES measurement methods may result in high heterogeneity and undermine the comparability of studies. To our knowledge, this is the first systematic review and meta-analysis on the topic.
The study will have wider representativeness by including individuals of all age groups and from all continents.

\section{Author affiliations}

${ }^{1}$ Department of Community Nutrition, School of Nutritional Sciences and Dietetics, Tehran University of Medical Sciences-International Campus (TUMS-IC), Tehran, the Islamic Republic of Iran

${ }^{2}$ Department of Epidemiology, University Medical Center Groningen, Groningen University, Groningen, The Netherlands

${ }^{3}$ Department of Nursing, Debre Berhan University, Debre Berhan, Ethiopia

${ }^{4}$ Department of Community Nutrition, School of Nutritional Sciences and Dietetics, Tehran University of Medical Science, Tehran, the Islamic Republic of Iran ${ }^{5}$ Obesity and Eating Habits Research Center, Endocrinology and Metabolism Molecular Cellular Sciences Institute, Tehran University of Medical Sciences, Tehran, the Islamic Republic of Iran

${ }^{6}$ Food Security Research Center, Department of Community Nutrition, Isfahan University of Medical Sciences, Isfahan, the Islamic Republic of Iran

Contributors SHM conceived and led the study. SHM, MMB, TDH, BST, TAS and AE developed search strategies and wrote the protocol. AE critically reviewed the final manuscript. All the authors read, commented, and approved the final manuscript.

Competing interests None declared.

Patient consent This study is based on extracting data from published studies.

Provenance and peer review Not commissioned; externally peer reviewed.

Open Access This is an Open Access article distributed in accordance with the Creative Commons Attribution Non Commercial (CC BY-NC 4.0) license, which permits others to distribute, remix, adapt, build upon this work non-commercially, and license their derivative works on different terms, provided the original work is properly cited and the use is non-commercial. See: http://creativecommons.org/ licenses/by-nc/4.0/

(c) Article author(s) (or their employer(s) unless otherwise stated in the text of the article) 2017. All rights reserved. No commercial use is permitted unless otherwise expressly granted.

\section{REFERENCES}

1. World Health Organization. Obesity and overweight fact sheet. 2014 http://www.who.int/mediacentre/factsheets/fs311/en/ (accessed 29 Apr 2017).

2. Ng M, Fleming $\mathrm{T}$, Robinson $\mathrm{M}$, et al. Global, regional, and national prevalence of overweight and obesity in children and adults during 1980-2013: a systematic analysis for the Global Burden of Disease Study 2013. Lancet 2014;384:766-81.

3. GBD 2015 Risk Factors Collaborators. Global, regional, and national comparative risk assessment of 79 behavioural, environmental and occupational, and metabolic risks or clusters of risks, 1990-2015: a systematic analysis for the Global Burden of Disease Study 2015. Lancet 2016;388:1659-724.

4. National Institute for Health and Environment (RIVM). Higher incidence of obesity and high blood pressure in new adult generations. 2016 http://www.rivm.nl (accessed 23 Apr 2017).

5. Alleyne G, Binagwaho A, Haines A, et al. Embedding noncommunicable diseases in the post-2015 development agenda. Lancet 2013;381:566-74.

6. Sallis JF, Owen N, Fisher EB. Ecological models of health behavior. In: Glanz K, Rimer B, Viswanath K, Health behavior and health education: theory, research, and practice. 4th ed. San Francisco: Jossey-Bass, 2008:464-85.

7. Papas MA, Alberg AJ, Ewing R, et al. The built environment and obesity. Epidemiol Rev 2007;29:129-43.

8. Ding D, Gebel K. Built environment, physical activity, and obesity: what have we learned from reviewing the literature? Health Place 2012;18:100-5.

9. Popkin BM, Duffey K, Gordon-Larsen P. Environmental influences on food choice, physical activity and energy balance. Physiol Behav 2005;86:603-13.

10. Maier W, Scheidt-Nave C, Holle R, et al. Area level deprivation is an independent determinant of prevalent type 2 diabetes and obesity at the national level in Germany. Results from the National Telephone Health Interview Surveys 'German Health Update' GEDA 2009 and 2010. PLoS One 2014;9:e89661. 
11. Powell-Wiley TM, Cooper-McCann R, Ayers C, et al. Change in neighborhood socio-economic status and weight gain: Dallas Heart Study. Am J Prev Med 2015;49:72-9.

12. Powell-Wiley TM, Ayers C, Agyemang P, et al. Neighborhood-level socio-economic deprivation predicts weight gain in a multi-ethnic population: longitudinal data from the Dallas Heart Study. Prev Med 2014;66:22-7.

13. Navalpotro L, Regidor E, Ortega P, et al. Area-based socioeconomic environment, obesity risk behaviours, area facilities and childhood overweight and obesity: socioeconomic environment and childhood overweight. Prev Med 2012;55:102-7.

14. Chetty R, Hendren N, Katz LF. The effects of exposure to better neighborhoods on children: new evidence from the moving to opportunity experiment. Am Econ Rev 2016;106:855-902.

15. de Wit L, Luppino F, van Straten A, et al. Depression and obesity: a meta-analysis of community-based studies. Psychiatry Res 2010;178:230-5.

16. Roberto CA, Swinburn B, Hawkes C, et al. Patchy progress on obesity prevention: emerging examples, entrenched barriers, and new thinking. Lancet 2015;385:2400-9.
17. Stroup DF, Berlin JA, Morton SC, et al. Meta-analysis of observational studies in epidemiology: a proposal for reporting. JAMA 2000;283:2008-12.

18. Moher $D$, Shamseer $L$, Clarke $M$, et al. Preferred reporting items for systematic review and meta-analysis protocols (PRISMA-P) 2015 statement. Syst Rev 2015;4:1.

19. Wells G, Shea B, O'Connell D, et al. The Newcastle-Ottawa Scale (NOS) for assessing the quality of nonrandomised studies in metaanalyses, 2000

20. Egger M, Davey Smith G, Schneider M, et al. Bias in meta-analysis detected by a simple, graphical test. BMJ 1997;315:629-34.

21. Duval S, Tweedie R. Trim and fill: a simple funnel-plot-based method of testing and adjusting for publication bias in meta-analysis. Biometrics 2000;56:455-63.

22. Higgins JP, Thompson SG. Quantifying heterogeneity in a metaanalysis. Stat Med 2002;21:1539-58.

23. Nordic Cochrane Centre The Cochrane Collaboration. Review manager (RevMan) [computer program] Version 53. Copenhagen: The Nordic Cochrane Centre, The Cochrane Collaboration, 2014. 